\title{
Análisis dinámico de una excavación profunda contenida mediante pilotes anclados en la grava de Santiago
}

Dynamic analysis of a deep excavation supported by anchored piles in Santiago gravel

Fecha de entrega: 10 de diciembre 2018 Fecha de aceptación: 15 de marzo 2019

\section{Felipe Salas ${ }^{1}$, Estéban Sáez ${ }^{1,2}$, Carlos Ovalle ${ }^{3}$ y Juan Manuel Fernández ${ }^{4}$}

${ }^{1}$ Departamento de Ingeniería Estructural y Geotécnica, Pontificia Universidad Católica de Chile, Avda. Vicuña Mackenna 4860, 7820436, Macul, Chile, flsalas@uc.cl, esaez@ing.puc.cl

${ }^{2}$ Centro de Investigación para la Gestión Integrada del Riesgo de Desastres CIGIDEN, Edificio Hernán Briones, 3er Piso. Av. Vicuña Mackenna 4860, Macul, Chile

${ }^{3}$ Research Institute of Mining and Environment RIME UQAT-Polytechnique, Department of Civil, Geological and Mining Engineering, Polytecnique Montréal, Room A-356, 2900 Édouard Montpetit Blvd, Montreal, QC, H3T 1J4, Canada, carlos.ovalle@polymtl.ca

${ }^{4}$ Pilotes Terratest Chile, Alonso de Córdova 5151, oficina 1401, Las Condes, Santiago, Chile, jmfernandez@terratest.cl

El sistema de contención más común en Santiago de Chile para excavaciones profundas corresponde a pilas (o pilotes) discontinuos y arriostrados lateralmente mediante anclajes. Este sistema se ha comportado satisfactoriamente en condiciones tanto estáticas como sísmicas. Los reducidos desplazamientos laterales observados sugieren que los procedimientos de diseño y que los códigos vigentes son conservadores y que un mejor entendimiento del comportamiento fisico del problema podría ayudar a optimizar las metodologías de diseño. Este trabajo expone los resultados de un análisis numérico en elementos finitos para un caso estático y dinámico, entregando una caracterización de incrementos de empujes sísmicos, desplazamientos postsísmicos permanentes y esfuerzos internos inducidos en los pilotes. Para esto se utilizó el modelo constitutivo HS-Small, calibrado en base a resultados de exploraciones geofísicas en terreno y de ensayos de laboratorio usando la técnica de gradación paralela. La respuesta dinámica fue estudiada para una gama de registros chilenos de aceleraciones, ajustados con el fin de ser representativos con los códigos de diseño vigentes en el país. A partir de estos resultados, se exponen conclusiones y recomendaciones acerca de los actuales procedimientos de diseño.

Palabras clave: modelación numérica, análisis dinámico, modelo HS-Small, esfuerzos internos
Discontinuous anchored piling support is one of the most frequently used retaining systems in temporary deep excavations in Santiago, Chile. The system has presented satisfactory behaviour under static and seismic conditions, with negligible deformations. These observations suggest that the design procedures and the local code requirements are conservative and a better understanding of the physical behaviour would lead to optimizing the design methodologies. This paper presents a static and dynamic analysis of a monitored pre-excavated pile-supported deep excavation. A finite element model was developed and calibrated based on static displacement measurements, geophysical and laboratory characterization using the parallel gradation technique. The dynamic responses to earthquake induced lateral pressures, induced internal forces and post-earthquake permanent displacement were studied for a suite of Chilean ground motions, scaled to be representative of current seismic design codes. Based on these results, some conclusions and recommendations on designing procedures are provided.

Keywords: numerical modelling, dynamic analysis, model HS-Small, internal forces

\section{Introducción}

La grava de Santiago es conocida por su alta competencia geotécnica y por presentar una cota de nivel freático por debajo de los $50 \mathrm{~m}$ de profundidad. Estas condiciones promueven el uso de pilas discontinuas ancladas como 
sistema de contención en excavaciones temporales. Por otra parte, Chile se caracteriza por ser un país con alta actividad sísmica, lo que motiva a estudiar en detalle una verificación sísmica que ayude a evitar daños en estructuras vecinas ante la eventual ocurrencia de un sismo significativo.

Usualmente, el diseño sísmico de una excavación temporal se realiza utilizando métodos pseudo-estáticos donde los corrimientos o desplazamientos horizontales permanentes $\left(\Delta_{\mathrm{eq}}\right)$ inducidos por un sismo, se relacionan implícitamente con el coeficiente pseudo-estático $\left(k_{\mathrm{h}}\right)$ escogido (Richards y Elms, 1979). La norma chilena NCh433 (2009) divide el país en 3 zonas de acuerdo a la máxima aceleración efectiva $\left(A_{0}\right)$ esperada, siendo este parámetro representativo a un evento sísmico con $10 \%$ de probabilidad de excedencia en 50 años con un período de retorno de 475 años (Riddell, 1995). La ciudad de Santiago pertenece a la zona II, lo que implica una $A_{0}$ igual a $0.3 \mathrm{~g}$. Las cargas sísmicas inducidas se incorporan mediante un $k_{\mathrm{h}}$ calculado en algunos casos como una fracción de $A_{0}$ (Manual de Carreteras, 2008), o en otros casos dependiendo un valor de $\Delta_{\mathrm{eq}}$ definido según la importancia de las estructuras vecinas, variando $\Delta_{\mathrm{eq}}$ entre 5 y 40 mm según el código chileno NCh3206 (2010).

Con el fin de analizar este actual procedimiento de cálculo, se estudió una excavación (ejecutada y monitoreada por Pilotes Terratest S.A. Chile) ubicada en la comuna de Las Condes, Santiago. La excavación alcanzó una profundidad de $21.2 \mathrm{~m}$ y fue contenida mediante pilotes pre-excavados con máquina y dos líneas de anclajes temporales. Uno de los pilotes fue instrumentado mediante un inclinómetro capaz de entregar un perfil de deformaciones horizontales en profundidad en las distintas etapas constructivas de la excavación (ver Figura 1).

El sitio se caracterizó geotécnicamente mediante métodos geofísicos activos y pasivos (MASW y ESPAC), y mediante ensayos de laboratorio a muestras remodeladas sobre material extraído en terreno. Debido a que el tamaño de las partículas que componen el terreno es demasiado grande para ser ensayado en equipos convencionales de laboratorio, se utilizó la técnica de gradación paralela (o curvas homotéticas) procurando conservar la representatividad de la muestra original en términos de resistencia al corte y dilatancia, limitando el contenido de finos a 9\% (de la Hoz, 2007). Se ejecutaron 2 ensayos triaxiales monótonos drenados (CID) a diferentes confinamientos, cuyos resultados fueron comparados con ensayos realizados in situ, a gran escala, a $3.5 \mathrm{~km}$ de la excavación estudiada (Kort et al., 1979). Una vez que se verificó la representatividad del material escalado en trayectorias triaxiales, se ejecutaron ensayos de corte cíclico en la muestra homotética para lograr obtener curvas de degradación de rigidez y amortiguamiento del material. Basados en esta caracterización, se calibró un modelo bidimensional en elementos finitos utilizando el software Plaxis ${ }^{\circledR}$. Estos resultados permitieron establecer conclusiones y recomendaciones para el proceso actual de diseño.

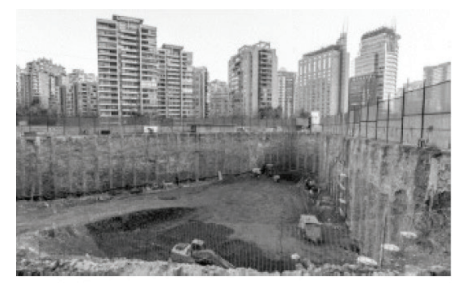

a)

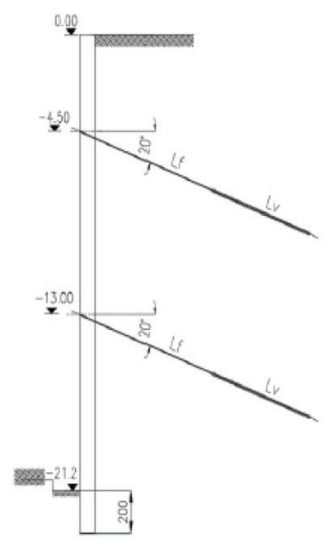

c)

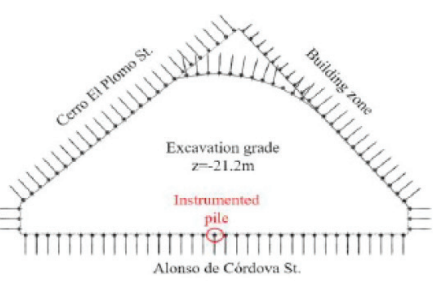

b)

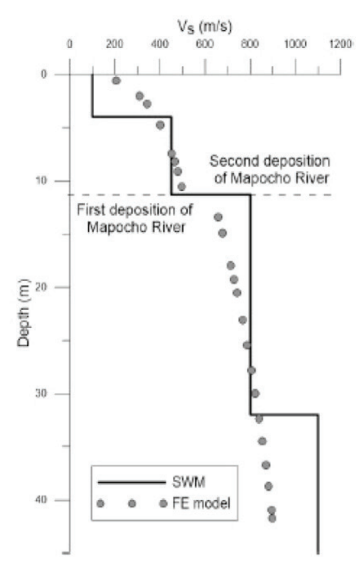

d)
Figura 1: Caso de estudio: a) fotografía de la excavación, b) plano en planta del proyecto, c) sección del pilote instrumentado y d) perfil de velocidad de corte $V_{\mathrm{s}}$ en profundidad

\section{Geometría de la excavación, mediciones en terreno y caracterización del sitio}

El sitio estudiado se caracteriza por presentar gravas areno-arcillosas bien graduadas (GW-GC), de compacidad alta y con partículas de más de $90 \mathrm{~mm}$ de tamaño máximo. El contenido de finos varía entre 2-3\% con un índice de plasticidad entre 5 y 30 . La napa freática se ubica a $50 \mathrm{~m}$ de profundidad. Esta unidad geológica es de origen fluvial y se conoce como deposiciones del río Mapocho. Basados en la exploración geofísica fue posible distinguir 2 estratos: la 
segunda depositación, desde la cota cero hasta los $11 \mathrm{~m}$ de profundidad, y la primera depositación, donde se alcanzó una velocidad de propagación de onda de corte cercana a $900 \mathrm{~m} / \mathrm{s}$ (roca) a los $33 \mathrm{~m}$ de profundidad, (ver Figura 1d). Ambos estratos varían entre sí primordialmente en su grado de compacidad y cohesión.

Para la contención de la excavación se utilizaron pilotes de hormigón armado, pre-excavados con máquina, de diámetro $880 \mathrm{~mm}$ y espaciados a $3.2 \mathrm{~m}$ eje a eje. Estos fueron arriostrados mediante dos líneas de anclajes provisorios ubicados a la cota -4.5 y $-13.0 \mathrm{~m}$, la primera y segunda línea respectivamente (Figura 1c). La primera línea fue tensada a una carga de bloqueo de $880 \mathrm{kN}$, mientras que la segunda a $1245 \mathrm{kN}$.

El escalamiento de las muestras, según el método de las curvas homotéticas, se hizo utilizando como base el material ensayado por Kort et al. (1979). Para ello se extrajo muestras de suelo a una profundidad de $10 \mathrm{~m}$. El proceso de escalamiento se realizó procurando conservar el coeficiente de uniformidad $\left(C_{\mathrm{u}}\right)$ y de concavidad $\left(C_{\mathrm{c}}\right)$ del material base, además de verificar que las densidades relativas (densidad máxima y mínima) se mantuvieran constantes (de la Hoz, 2007). El contenido de finos se limitó a un $9 \%$, con el fin de que la matriz fina no dominara en la resistencia al corte ni dilatancia del material (Rollins et al., 1998).

Se ejecutaron 2 ensayos triaxiales monótonos drenados (CID) a las muestras escaladas utilizando los mismos confinamientos empleados por Kort et al. (1979) en sus ensayos a gran escala (44 y $83 \mathrm{kPa}$ ) de acuerdo a ASTM D5311 (2013). Se procuró confeccionar las probetas a la misma densidad in situ del terreno. Una vez logrado un correcto ajuste entre ambas curvas (material original a gran escala y material escalado), se ejecutaron ensayos cíclicos en probetas de $5 \times 10 \mathrm{~cm}$ (torsión cíclica y columna resonante) completando la caracterización del material. Las curvas de degradación obtenidas fueron comparadas con curvas de ensayos a gran escala y al mismo confinamiento para gravas (Rollins et al., 1998). Los resultados en términos de degradación de rigidez son muy similares a los reportados por otros autores, mientras el amortiguamiento observado es un poco superior al de la literatura si la deformación angular es inferior al $0.003 \%$.

\section{Modelo de elementos finitos en Plaxis} Parámetros de modelación del suelo

Se utilizó el modelo constitutivo Hardening Soil with Small Strains (HS-Small) para la modelación de ambos estratos de suelo. Este modelo es una extensión de la formulación matemática del modelo Hardening Soil (HS) que presenta una dependencia no-lineal hiperbólica para la relación esfuerzo-deformación (Duncan y Chang, 1970), además de incorporar endurecimiento tanto en trayectorias isotrópicas como de corte, lo que ayuda a predecir de manera más realistas las deformaciones inducidas. El modelo HS-Small ofrece la posibilidad de incorporar un valor de rigidez al corte a muy pequeñas deformaciones $\left(G_{\max }\right)$ y degradación no-lineal ante ciclos de cargadescarga (Hardin y Drnevich, 1972).

Mediante la herramienta de Plaxis ${ }^{\circledR}$ Soil-test se simularon los ensayos efectuados en laboratorio, buscando el mejor ajuste entre las curvas de esfuerzo-deformación y curvas de degradación reales con las modeladas (Figura 2). Para complementar esta calibración de parámetros, se utilizaron los resultados de geofísica cuya curva simulada se expone en la Figura 1d. Los parámetros obtenidos se presentan en la Tabla 1. a)

c)

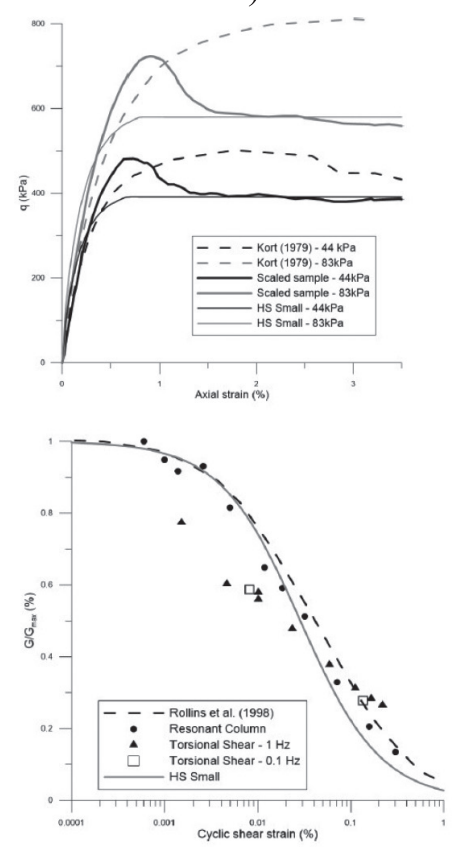

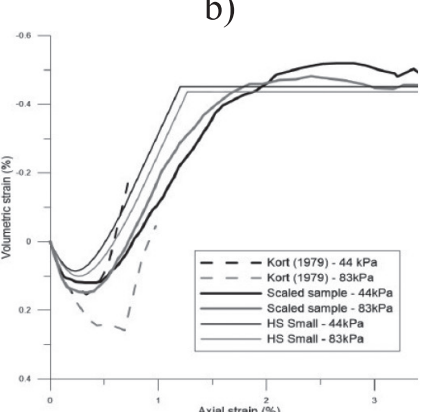

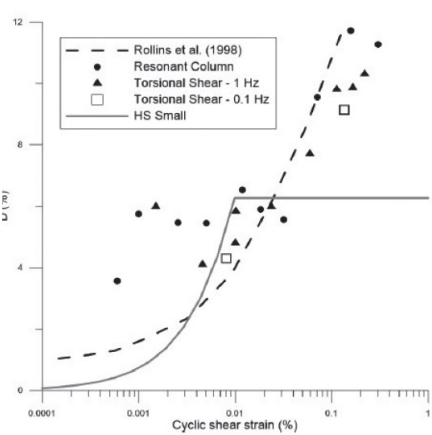

d)
Figura 2: (a) y (b) Comparación entre curvas de ensayo triaxial CID: material original (Kort et al., 1979), escalado, y simulaciones hechas en Plaxis según HS-Small. (c) y (d) Resultados de ensayos de corte cíclico: material escalado, curvas de referencia (Rollins et al., 1998) y simulaciones según HSSmall 
Tabla 1: Propiedades para las capas de suelo usando el modelo HS small

\begin{tabular}{|c|c|c|c|}
\hline \multirow{2}{*}{ Parámetros } & \multirow{2}{*}{ Unidad } & \multicolumn{2}{|c|}{ Estratos de suelo } \\
\cline { 3 - 4 } & & $\begin{array}{c}\text { Primera } \\
\text { depositación }\end{array}$ & $\begin{array}{c}\text { Segunda } \\
\text { depositación }\end{array}$ \\
\hline$\gamma^{\prime}$ & $\mathrm{kN} / \mathrm{m}^{3}$ & 22.5 & 22.0 \\
\hline $\mathrm{c}^{\prime}$ & $\mathrm{kN} / \mathrm{m}^{2}$ & 35.0 & 15.0 \\
\hline$\phi^{\prime}$ & $\circ$ & 45.0 & 45.0 \\
\hline$\psi$ & $\circ$ & 15.0 & 10.0 \\
\hline$m$ & - & 0.51 & 0.55 \\
\hline$E_{50}^{\text {ref }}$ & $\mathrm{MN} / \mathrm{m}^{2}$ & 275.0 & 80.0 \\
\hline$E_{\text {oed }}^{\text {ref }}$ & $\mathrm{MN} / \mathrm{m}^{2}$ & 190.0 & 130.0 \\
\hline$E_{\text {ur }}$ & $\mathrm{MN} / \mathrm{m}^{2}$ & 900.0 & 350.0 \\
\hline$G_{\max }$ & $\mathrm{MN} / \mathrm{m}^{2}$ & 750.0 & 400.0 \\
\hline$\gamma_{0.7}$ & - & 0.0011 & 0.0008 \\
\hline$v_{\text {ur }}$ & - & 0.2 & 0.3 \\
\hline$K_{0}^{\text {nc }}$ & - & 0.43 & 0.65 \\
\hline
\end{tabular}

\section{Elementos estructurales}

Los pilotes pre-excavados fueron modelados utilizando elementos tipo Plate elasto-plásticos según diseño a flexocompresión, incorporando el espaciamiento entre pilotes de $3.2 \mathrm{~m}$ indirectamente en los parámetros de rigidez axial y flexural. Alrededor de los pilotes se definieron interfaces de suelo-estructura cuyo criterio de ruptura obedece al criterio de Mohr-Coulomb. La longitud libre de los anclajes fue modelada mediante elementos Node-to-Node Anchors donde la carga de tensado fue aplicada conforme a la secuencia constructiva. La longitud de bulbo de los anclajes fue modelada mediante elementos Embeddedpile row con el fin de lograr una mejor representación del traspaso de carga entre el bulbo y el suelo.

\section{Modelo}

Se desarrolló un modelo bi-dimensional en deformaciones planas utilizando el software Plaxis ${ }^{\circledR}$, como se muestra en la Figura 3. Se consideró una profundidad del modelo de $80 \mathrm{~m}$ con el fin de representar roca elástica (Elastic bedrock) en su base, según el perfil de velocidad de ondas de corte obtenido (Figura 1d). La extensión lateral del modelo fue de $115 \mathrm{~m}$, definida con el objetivo de satisfacer condiciones de campo libre, es decir, lo suficientemente lejos de la perturbación generada por la excavación. En la fase estática se utilizaron bordes con restricción del movimiento en la dirección normal a estos, mientras que para la fase dinámica se emplearon elementos Free-field (o campo libre) en los límites laterales y Compliant base en el extremo inferior. Esto último con el fin de evitar la reflexión de las ondas sísmicas dentro del modelo. Se utilizaron elementos de 15 nodos cuyo tamaño fue escogido para garantizar un mínimo de 8 elementos por longitud de onda, considerando un contenido de frecuencia relevante desde 0 hasta $15 \mathrm{~Hz}$ y un $V_{\mathrm{s}, 30}=600 \mathrm{~m} / \mathrm{s}$.

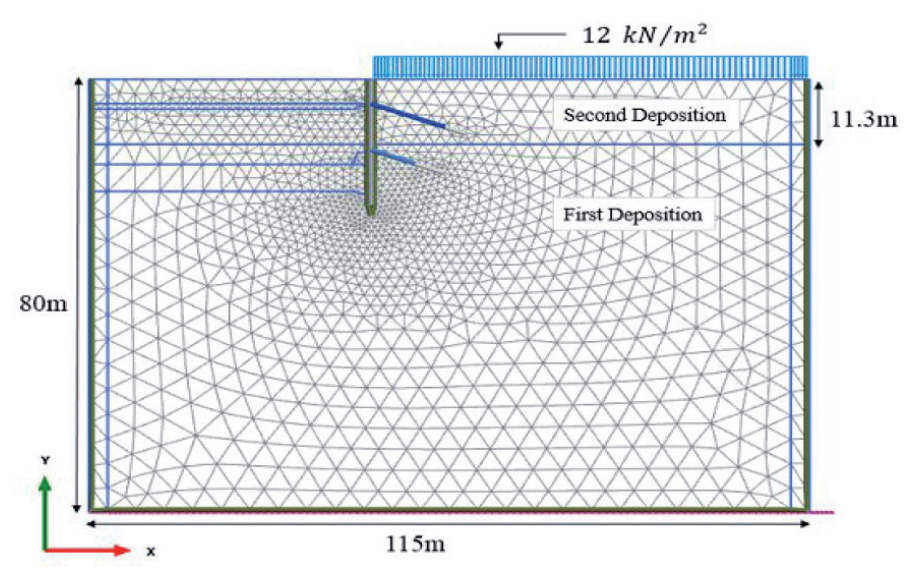

Figura 3: Geometría de modelo de EF en 2D

\section{Selección y calibración de input dinámico}

Uno de los aspectos claves para llevar a cabo un análisis dinámico, es la elección y calibración de una solicitación dinámica representativa del sitio estudiado. Esta elección es complicada debido a la alta variabilidad entre los distintos eventos sísmicos. Una posible solución es considerar una amplia gama de registros de aceleraciones. En este estudio se analizaron registros medidos en los terremotos del Maule $2010\left(M_{\mathrm{w}}=8.8\right)$ y de Valparaíso $1985\left(M_{\mathrm{w}}=8.0\right)$, todos medidos en roca (outcropping rock). Estos registros fueron escalados e impuestos en la base del modelo. El escalamiento consistió en amplificar iterativamente el registro de aceleraciones de tal forma de obtener una $A_{0}$ $=0.3 \mathrm{~g}$ en superficie. De esta manera se aseguró que el input dinámico impuesto fuese representativo con lo que establece la norma chilena NCh433 (2009). Para mayores detalles acerca de esta calibración y los distintos espectros de respuesta obtenidos, revisar Salas (2018).

\section{Resultados}

\section{Análisis estático}

Para el análisis estático se consideraron 8 etapas diferentes, comenzando por una etapa de inicialización de esfuerzos 
empleando el método de cálculo Gravity loading de Plaxis ${ }^{\circledR}$ para garantizar el equilibrio de esfuerzos en el modelo. Luego de esta etapa se activó la sobrecarga equivalente representativa de calles, igual a $12 \mathrm{kPa}(\mathrm{NCh} 3206,2010)$. Como tercera etapa se modeló la ejecución e instalación de los pilotes y activación de los elementos de interfaz. Luego se respetó el procedimiento de ejecución de los anclajes, el cual consiste en excavar $0.5 \mathrm{~m}$ por debajo de la cota de cada anclaje, instalar el cable y tensarlo a la carga de bloqueo estipulada. Se repitió esta secuencia hasta alcanzar el nivel de sello de fundación $(21.2 \mathrm{~m})$.

Las Figuras 4a y 4b muestran una comparación entre los resultados obtenidos mediante el modelo numérico y lo medido en terreno mediante un inclinómetro en distintas etapas. Se observa un buen ajuste entre ambas curvas, alcanzando un valor máximo de deflexión de $15 \mathrm{~mm}$ en la etapa final de la excavación. Se concluye que el modelo es capaz de reproducir apropiadamente las deformaciones producidas en los pilotes inducidas por la excavación. La Figura 4c muestra la distribución de empujes calculada en la última etapa del análisis estático y es comparado con el diagrama de empujes de diseño empleado y con perfiles teóricos de empujes (activo de Rankine y geostático). Se concluye que en la medida en que el pilote se deforma, se produce una relajación de esfuerzos disminuyendo los empujes horizontales que actúan sobre el mismo. Se observa además una concentración de esfuerzos en las cotas de anclaje, esto es debido al tensado de los mismos.

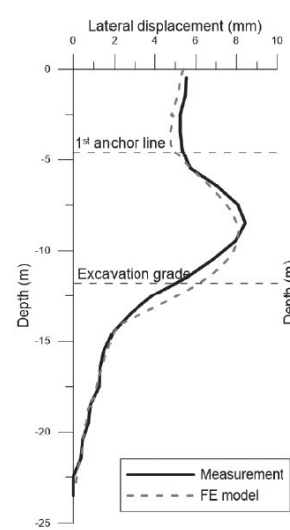

a)

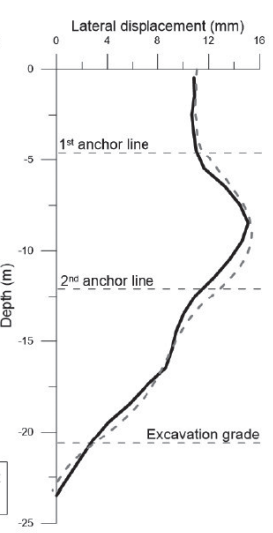

b)

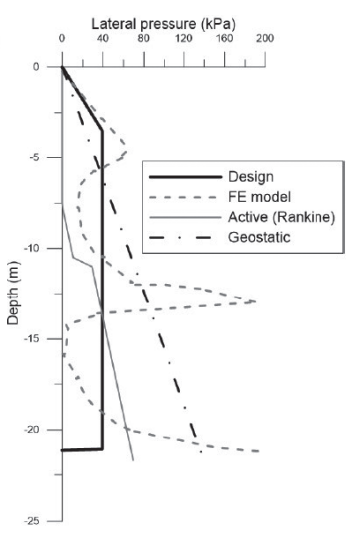

c)
Figura 4: a) Comparación entre desplazamientos horizontales medidos con inclinómetros y calculados con modelo de EF a una profundidad intermedia de excavación, b) profundidad final de excavación y c) perfiles de empujes laterales en la etapa final de la excavación: distribución geoestática, de Rankine, de diseño y resultados de modelo de EF

\section{Análisis dinámico}

Se monitorearon los desplazamientos de la primera línea de anclajes $\Delta_{\text {eq }}$ según la NCh3206 (2010) durante todo el evento sísmico en el modelo de EF, obteniendo un diagrama tiempo-historia de dichas deformaciones. La Figura 5 muestra este diagrama para los distintos registros empleados en sus 2 componentes (NS y EW). Se observa que se alcanza un máximo valor instantáneo de $79 \mathrm{~mm}$ para el registro medido en Las Tórtolas. Los desplazamientos residuales permanentes $\Delta_{\text {eq }}$ varían entre el rango de 10 a $20 \mathrm{~mm}$.
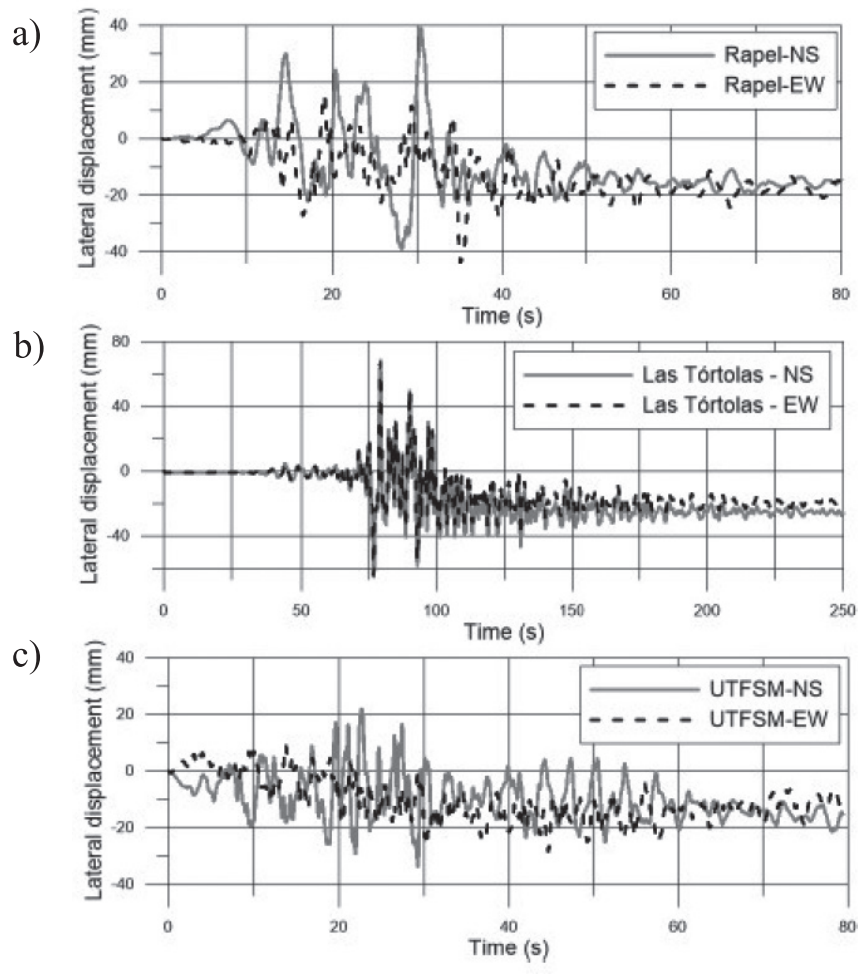

Figura 5: Diagramas tiempo-historia de desplazamienos del primer anclaje: a) registro de Rapel (Maule 2010), b) Las Tórtolas (Maule 2010) y c) UTFSM (Valparaíso 1985)

De cada uno de los registros utilizados, se registraron 10 perfiles instantáneos del incremento de empujes laterales inducidos por la solicitación sísmica durante la duración significativa con intensidad de Arias entre 5-95\% (Arias, 1970). Dado que la envolvente de estos 10 perfiles no representa un estado de carga envolvente actuando sobre el pilote, se calculó un promedio de estos 10 perfiles instantáneos los cuales se muestran en la Figura 6a con línea segmentada. Estos perfiles se comparan con recomendaciones de empujes para muros perfectamente arriostrados según el código NCh433 (2009), y a su 
vez con el empuje teórico de Mononobe-Okabe M-O (Mononobe y Matsuo, 1929) siguiendo una distribución triangular invertida (Seed y Whitman, 1970) utilizando $k_{\mathrm{h}}$ $=0.15 \mathrm{y} k_{\mathrm{v}}=0.0$. Se observa que el promedio de los perfiles instantáneos calculados no se ajusta a ninguna de las dos teorías, esto es debido a que la distribución que se propone en la NCh433 (2009) se utiliza en muros perfectamente arriostrados, por ejemplo, muros de subterráneos y no en pilotes que admiten deformación. Por otro lado, la teoría de $\mathrm{M}-\mathrm{O}$ asume un muro gravitacional, infinitamente rígido y deslizante. Situación muy distinta a la de un pilote arriostrado mediante anclajes.

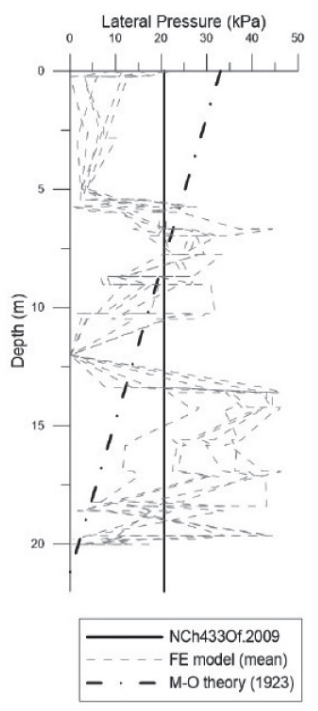

a)

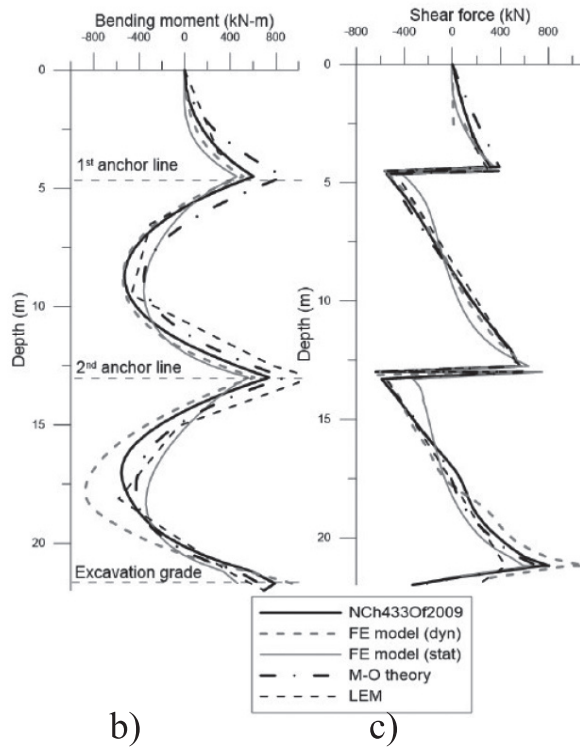

C)
Figura 6: Comparación entre metodologias descritas. a) Perfiles de incremento de empujes sísmicos, b) diagrama de momentos y c) diagrama de corte

Con respecto a los esfuerzos internos de los pilotes, se calculó una envolvente del diagrama de momento y de corte una vez terminada la etapa dinámica como se muestra en las Figuras $6 \mathrm{~b}$ y $6 \mathrm{c}$. Estos valores resumen la máxima respuesta dinámica observada en los pilotes, por lo que son estos valores los que deberían ser tomados en cuenta para el diseño estructural. Estos diagramas son comparados con el diagrama de esfuerzos estático obtenido y con diagramas de esfuerzos obtenidos después de aplicar la sobrecarga sísmica de la NCh433 (2009) y el empuje de M-O previamente descrito. El diseño de los pilotes se realizó mediante el software alemán GGURetain ${ }^{\circledR}$, el cual utiliza la metodología de equilibrio límite (LEM) para determinar el largo de los anclajes, en función de un factor de seguridad determinado (DIN4085, 2011; EAB, 2013). Los diagramas de esfuerzos internos luego de aplicar esta metodología también son expuestos. El valor máximo positivo obtenido del análisis dinámico alcanza los $937 \mathrm{kNm}$ en el nivel de sello de fundación, mientras que el máximo negativo es igual a $-875 \mathrm{kNm}$ y se alcanza entre la segunda línea de anclajes y el nivel de sello de fundación. Se obtiene un incremento sísmico de un 157\% en el diagrama de momento con respecto al perfil estático y de un $53 \%$ con respecto al diagrama obtenido según la NCh433 (2009). A esta profundidad el efecto en el diagrama de momento de la sobrecarga escogida para el método de $\mathrm{M}-\mathrm{O}$ es despreciable. El diagrama obtenido a partir de un análisis LEM muestra un máximo valor positivo de 1117 $\mathrm{kNm}$ en la segunda línea de los anclajes. Valor que es un $20 \%$ mayor al calculado mediante un análisis dinámico.

Con respecto a los diagramas de corte, se observa una tendencia similar a la de los momentos entre todas las curvas, obteniendo un máximo valor igual a $1098 \mathrm{kN}$, presentando un incremento sísmico de un $49 \%$ con respecto al valor estático.

Se observó que la evolución de carga en los anclajes durante las solicitaciones sísmicas, no superó un $14 \%$ de incremento con respecto al valor inicial (última fase de análisis estático).

\section{Conclusiones}

El presente artículo presenta una investigación cuyo objetivo fue caracterizar los incrementos de empujes laterales inducidos por un evento sísmico, los desplazamientos permanentes inducidos, y los esfuerzos internos sísmicos inducidos. Las principales conclusiones son:

(a) El método de las curvas homotéticas (o gradación paralela) es capaz de mantener la representatividad del material original en términos de resistencia al corte y dilatancia en deformaciones menores a un $0.3 \%$. Basados en esta metodología se propusieron curvas de degradación de rigidez y amortiguamiento para la grava de Santiago.

(b) El análisis dinámico llevado a cabo con registros previamente calibrados con el fin de ser representativos con la normativa vigente, muestra que los desplazamientos laterales permanentes obtenidos en la cabeza de la primera línea de anclaje 
se encuentran en el rango de 10 a $20 \mathrm{~mm}$.

(c) Se obtuvo un máximo momento sísmico de un $157 \%$ mayor con respecto al valor máximo obtenido en la etapa estática. Este valor es un $20 \%$ menor al calculado mediante una metodología de LEM, actual procedimiento de diseño.

(d) Se observó que la evolución de carga en los anclajes durante las solicitaciones sísmicas no superó un 14\% de incremento con respecto al valor inicial.

(e) Se concluye que el diseño de excavaciones profundas en áreas de alta sismicidad debe ir respaldado de un análisis dinámico con el fin de conocer con mayor exactitud las deformaciones sísmicas inducidas. Para el caso de estudio, este tipo de análisis hubiera permitido reducir las cargas sísmicas sobre las pilas.

\section{Agradecimientos}

Esta investigación fue parcialmente financiada por el Centro para la Gestión Integrada del Riesgo de Desastres (CIGIDEN) CONICYT/FONDAP/15110017. El primer autor también fue parcialmente financiado por Pilotest Terratest S.A.

\section{Referencias}

Arias, A. (1970). A measure of earthquake intensity. Hansej R.J. editor. Seismic design for nuclear power plants. Cambridge, USA

ASTM D5311 (2013). Standard test method for load controlled cyclic triaxial strength of soil. ASTM International, West Conshohocken, PA, USA

de la Hoz, K. (2007). Estimación de los parámetros de resistencia al corte en suelos granulares gruesos. Tesis MSc, Universidad de Chile, Santiago

DIN4085 (2011). Subsoil- calculation of earth pressure. Deutsches Institut für Normung, Berlin, Germany

Duncan, J.M. and Chang, C. (1970). Nonlinear analysis of stress and strain in soils. Journal of the Soil Mechanics and Foundation Division 96(5), 1629-1653

EAB (2013). Recommendations on excavations. German Geotechnical Society, Wiley, Germany
Hardin, B.O. and Drnevich, V.P. (1972). Shear modulus and damping in soils: measurement and parameter effects. Journal of the Soil Mechanics and Foundation Division 98(6), 603-624

Kort, I., Musante, H. and Fahrenkrog, C. (1979). In-situ mechanical properties measurements of gravelly soil used in an interaction and foundation model for the Santiago Metro. $6^{\text {th }}$ Panamerican Conference on Soil Mechanics and Foundation Engineering, Lima, Peru, vol. 2, 217-224

Manual de Carreteras (2008). Instrucciones y criterios de diseño. Volumen 3. Ministerio de Obras Públicas, Santiago, Chile

Mononobe, N. and Matsuo, H. (1929). On the determination of earth pressure during earthquakes. World Engineering Congress, Beijing, China, vol. 9, 177-185

NCh3206 (2010). Geotecnia- Excavaciones, entibaciones y socalzados- Requisitos. Instituto Nacional de Normalización INN, Santiago, Chile

NCh433 (2009). Diseño sísmico de edificios. Instituto Nacional de Normalización INN, Santiago, Chile

Richards, R.J. and Elms, D. (1979). Seismic behavior of gravity retaining walls. Journal of the Geotechnical Engineering Division 105(4), 449-464

Riddell, R. (1995). Inelastic design spectra accounting for soil conditions. Earthquake Engineering \& Structural Dynamics 24(11), 1491-1510

Rollins, K.M., Evans. M.D., Diehl, N.B. and Daily, W.D. (1998). Shear modulus and damping relationships for gravels. Journal of Geotechnical and Geoenvironmental Engineering 124(5), 396405

Salas, F. (2018). Monitoring and dynamic analysis of a discontinuous pile-supported deep excavation in Santiago gravel. Tesis MSc, Pontificia Universdiad Católica de Chile, Santiago.

Seed, H.B. and Whitman, R.V. (1970). Design of earth retaining structures for dynamic loads. ASCE Specialty Conference on Lateral Stresses in the Ground and Design of Earth Retaining Structures, vol. 1, Cornell University, Ithaca, NY, USA, 103-147 